\title{
Seroprevalence Trends of Leptospirosis at a Tertiary Care Centre of North India from 2016-2019 Determined by Two Commercial Elisa Kits
}

\author{
Sangram Singh Patel $(\mathbb{D}$, Sweta Singh* (D), Chinmoy Sahu (iD) and Hemant Verma \\ Department of Microbiology, Sanjay Gandhi Post Graduate institute of Medical Sciences (SGPGIMS), \\ Lucknow - 226 014, India.
}

\begin{abstract}
Leptospirosis is a worldwide zoonotic disease which remains under diagnosed in most of the regions of our country due to the lack of proper diagnostic facility and set up. Diagnosis usually depends on Microscopy,culture and serological tests like ELISA, MAT(Microscopic agglutination test ). MAT is the gold standard diagnostic test but it requires live leptospires and it can only be done in a reference laboratory. Hence, ELISA is the most preferred cost-effective serological method with both sensitivity and specificity of $95 \%$. The Aim of our present study was to evaluate the trends of seroprevalence of leptospirosis from January 2016 to December 2019 and to compare two ELISA kits for the serodiagnosis of Leptospirosis. The study was conducted between January 2016 to December 2019 and 1611 blood samples were collected from clinically suspected cases of leptospirosis. Detection of IgM antibodies to leptospira was done by DRG andRecombilisa (CTK) ELISA kits. Results by both the kits were analysed. Seventy samples were taken as controls from different patients on random basis to prevent any type of biasing. Samples were tested for other co-infections and Demographic parameters were studied. Seroprevalence was estimated to be $4.8 \%$ by DRG KIT and $5.5 \%$ by Recombilisa CTK kit. Average seroprevalence was noted to be $\mathbf{5 . 1 \%}$. The diagnosis of presumptive and possible Leptospirosis was made in $\mathbf{9 6}(78 \%)$ and $\mathbf{2 7}(22 \%)$ respectively, by modified Faine's criteria. The seroprevalence between 2016 to 2019 varied from $0.57-1.8 \%$. The months of July to November of year 2017 noted the highest prevalence. Out of these patients, majority (96\%) showed significant improvement during the follow-up period after 2-3 months with empirical treatment. Typhoid was seen as most common co- infection in these patients.

Keywords: Leptospira, ELISA, Microscopic agglutination test (MAT), seroprevalence, serological methods
\end{abstract}

*Correspondence: swetasinghkarn@gmail.com
(Received: February 26, 2020; accepted: May 09, 2020)
Citation: Patel SS, Singh S, Sahu C, Verma H. Seroprevalence Trends of Leptospirosis at a Tertiary Care Centre of North India from
2016-2019 Determined by Two Commercial Elisa Kits. J Pure App/ Microbiol. 2020;14(2):1263-1268. doi: 10.22207/JPAM.14.2.21
(C) The Author(s) 2020. Open Access. This article is distributed under the terms of the Creative Commons Attribution 4.0 International License which
permits unrestricted use, sharing, distribution, and reproduction in any medium, provided you give appropriate credit to the original author(s) and
the source, provide a link to the Creative Commons license, and indicate if changes were made. 


\section{INTRODUCTION}

Leptospirosis is a worldwide zoonotic disease caused by a pathogenic spirochaete belonging to the genus, Leptospira ${ }^{1}$. Rats are the most common reservoirs of infection and are involved in transmission, either directly or indirectly by contaminating water and soil with their urine. The clinical manifestations of Leptospirosis range from mild catarrh-like illness to icteric disease with severe liver and kidney involvement ${ }^{2,3}$. Organism enters the host through skin abrasions, mucosal surfaces or the eye. Incubation period ranges from 3 to 30 days; usually 10- 12 days.

The disease remains underdiagnosed in most of the regions of India due to the lack of proper diagnostic facility and set up $^{4}$. Diagnosis usually depends on Microscopy, culture and serological tests like ELISA, MAT(Microscopic agglutination test). Microscopic agglutination test (MAT) is the gold standard test and is serogroup specific. However, it requires live leptospires and also complex in performance, so this test always requires a reference laboratory. IgM ELISA is a good alternative serological test and is genus specific ${ }^{5}$. It allows the detection of antibody in the 1st week of illness with sensitivity ranging from $68 \%$ to $100 \%$ and specificity up to $97 \%$. ELISA is the most preferred cost-effective serological method with both sensitivity and specificity of $95 \%$.

The aim of our present study was to evaluate the trends of seroprevalence of leptospirosis from January 2016 to December 2019 and to compare the results of two commercial ELISA kits.

\section{MATERIALS AND METHODS Clinical samples}

The study was conducted at the department of Microbiology, Sanjay Gandhi Postgraduate Institute of Medical Sciences, Lucknow between January 2016 to December 2019. The study was approved by the Institute's ethical committee. A total of 1611 blood samples were collected from clinically suspected cases of leptospirosis attending either outpatient departments or were admitted in the Gastroenterology, Neurology, Paediatrics, surgery or Gynaecology wards, etc. and were analysed retrospectively.
Diagnosis of presumptive and possible Leptospirosis was done by modified FAINE'S Criteria $^{6,7}$. Inclusion criteria was all Patients who were suspected clinically of leptospirosis and presented with a history of fever for $>7$ days accompanied with any of the following features i.e., severe headache, myalgia, arthralgia, conjunctival suffusion, uveitis, rash, hepatosplenomegaly, hemorrhagic manifestations, renal failure, aseptic meningitis, Acute respiratory distress syndrome ${ }^{7}$. Serological testing protocol

Five $\mathrm{ml}$ of venous blood was collected in a plain vial without anticoagulant and serum was separated after centrifugation at $3000 \mathrm{rpm}$ for 5 minutes. This serum was used for further testing. Detection of IgM antibodies to leptospira was simultaneously done by DRG ELISA kit (Inc. USA) and Recombilisa ELISA kit (CTK Biotech, Inc. California). They are qualitative enzyme immunoassays for the detection of antibodies to Leptospira biflexa in samples of human serum or plasma. Seventy samples were taken as controls from different patients randomly and were tested by both the ELISA kits.

\section{Interpretation of ELISA Results DRG Kit}

Non-reactive $=0.0-0.15$ OD units;

Reactive $>1.0$ OD.

Sample OD between 0.15-1.00 = No interpretation as per the Kit. These samples were taken as Equivocal in our study.

\section{Recombilisa CTK Kit}

Cut-off value $=0.244+$ Negative control

OD. off value.

Specimen OD Ratio $=$ Specimen OD $/$ Cut -

Negative $<1.00$ specimen OD ratio

Positive $>=1.00$ specimen $\mathrm{OD}$ ratio

Testing for other co-Infections

Samples were tested for other infections in the following manner:

Malaria by SD Bioline rapid test kit for $P$. falciparum and P. vivax

Typhoid by Widaltest for $\mathrm{TO}, \mathrm{TH}, \mathrm{AH}, \mathrm{BH}$

titres

Dengue by Panbio ELISA kit

Chikungunya by SD and NIV ELISA kit.

Scrub Typhus by InBios ELISA kit for IgM

detection. 


\section{Statistical Analysis}

Data was analyzed using the SPSS (Statistical Package for Social Sciences) software for descriptive statistics, and the results were expressed in proportions, mean, and standard deviation. Categorical data were described using numbers and percentages. Statistical significance was calculated by $P$ value.

\section{RESULTS}

A total of 1611 patients were enrolled in this study of over 4 years time period. All the serum samples were simultaneously tested by both the ELISA kits for comparison.

Out Of 1611 samples, 77 were positive for anti-leptospira IgM antibodies with OD values > 1.00; whereas 46 samples tested as Equivocal with OD values between $0.540-8.50$ by DRG ELISA kit. Rest of the samples were negative with OD values below 0.15 . Seroprevalence was calculated as $4.8 \%$ by DRG kit.

Out of 1611 samples, 89 tested positive with specimen $O D$ ratio $>1.00$. Rest of the samples were found to be negative with specimen $O D$ ratio $<1.00$. Seroprevalence was calculated as $5.5 \%$ by CTK RECOMBILISA kit.(Fig. 1)

All the 70 control samples were tested as negative by both the ELISA kits with OD values between $0.12-0.22$. These samples were
Table 1. Distribution of Signs \& Symptoms in the patients

\begin{tabular}{lc}
\hline Signs \& Symptoms & Patients (\%) \\
\hline Fever & 100 \\
Jaundice & 89 \\
Myalgia & 88 \\
Headache & 76 \\
Chills \& rigors & 52 \\
Arthritis \& Arthralgia & 42 \\
Hepatomegaly & 36 \\
Oliguria & 34 \\
Abdominal pain & 33 \\
Splenomegaly & 25 \\
Cough & 23 \\
Conjunctival suffusion & 19 \\
Renal involvement & 14 \\
\hline
\end{tabular}

also tested for other infections like Malaria, Chikungunya, Typhoid, Scrub typhus and Dengue. Among these; 11 samples tested positive for Typhoid, 7 for malaria, 4 for dengue, 2 for chikengunya and only 1 for scrub typhus.(Fig.2).

The diagnosis of presumptive and possible Leptospirosis was made in $96(78 \%)$ and $27(22 \%)$ respectively, by modified Faine's criteria. The seroprevalence between 2016 to 2019 varied from $0.57-1.8 \%$. The months of July to November of year 2017 noted the highest prevalence; confirming seasonal prevalence of

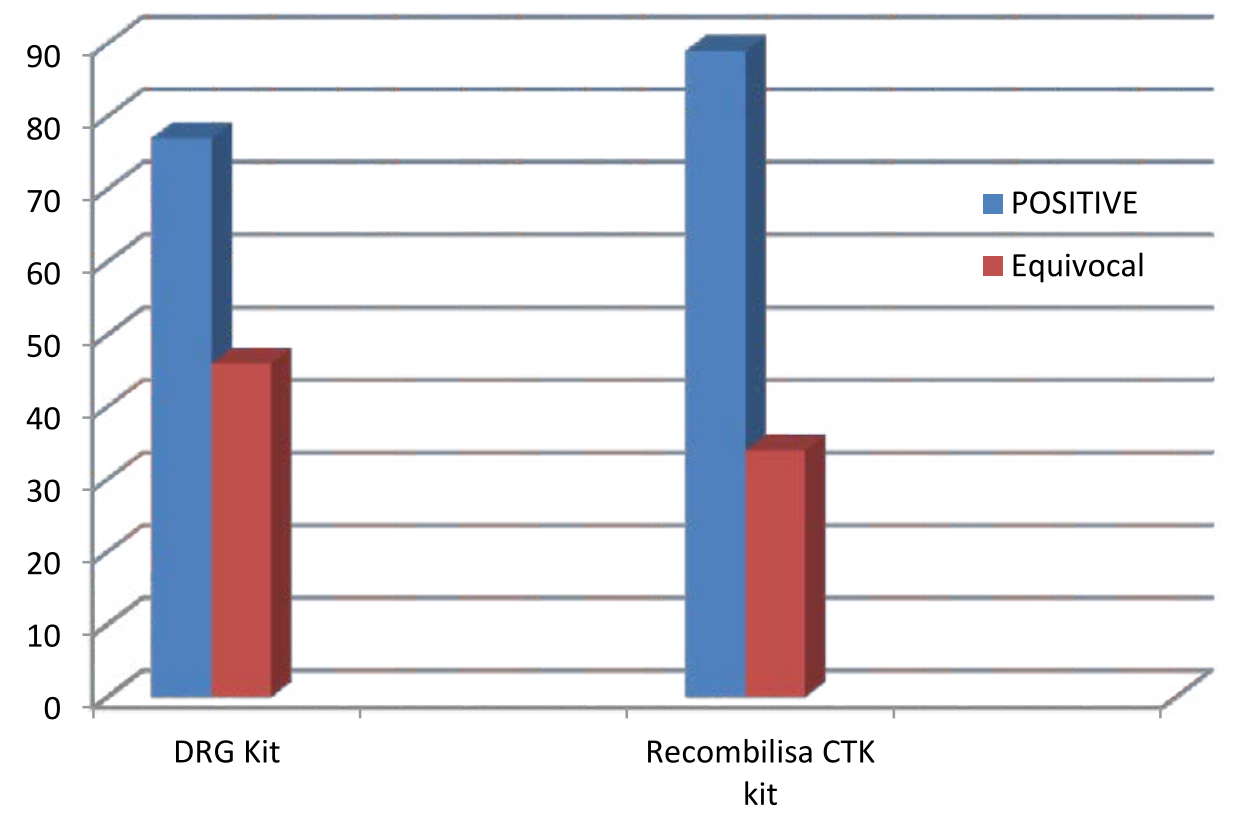

Fig. 1. Comparison of DRG and Recombilisa CTK ELISA kits. 


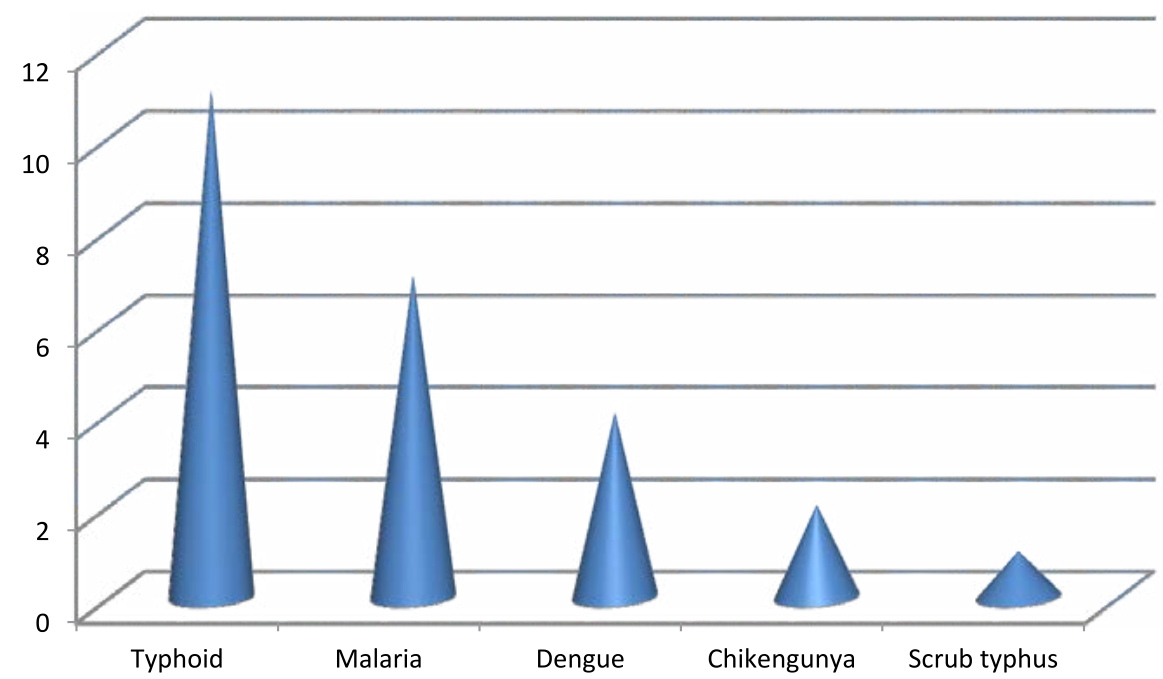

Fig. 2. Co-infections observed in patients of Leptospirosis.

leptospirosis in these rainy seasons. Amongthe 123 positive samples, 95 (77\%) were males and 28 (23\%)females; with male: female ratio of 3.39:1. Age of the patients ranged from 8 to 82 years. The most common presenting features were fever, myalgia and jaundice and was seen in almost $88 \%$ of the patients (Table 1).

\section{Follow-up of the Patients}

Out of these 84 patients, 79 were given empirical treatment for leptospirosis and showed significant improvement during the follow-up period after 2-3 months. Out of the remaining 5 cases, 2 required dialysis; whereas; 3 suffered mortality due to renal failure which was attributable to other comorbid conditions in these patients.

\section{DISCUSSION}

The present study evaluated the seroprevalence of leptospirosis along with the associated epidemiological risk factors and compared two ELISA kits for serodiagnosis of leptospirosis.

Seroprevalence was estimated to be $5.1 \%$ in our study. The seropositivity observed in our hospital was much lower as compared to other similar studies which was $18-38 \%^{8,9}$. However, our study seroprevalence rates correlated well with a similar study done at AIIMS, New Delhi with a seroprevalence rate of $6.4 \%{ }^{10}$. This decrease in seroprevalence compared to other studies may be attributed to several factors such as increase in awareness, improved hygienic practices, use of safe drinking water, and better socioeconomic condition. The DRG kit appeared to produce false negative results with twelve sera. Four were from confirmed cases of leptospirosis with no further samples included in this study. Three were from convalescent samples taken five months afteronset, an earlier sample was positive by CTK KIT. These twelve sera were positive by CTK recombilisa kit. The occasional false negative results seen with DRG kit indicates that paired serum samples must be examined from suspected clinical cases before a diagnosis of leptospirosis is made. The sensitivity and specificity of the kits as mentioned in their literature was: DRG KIT 90\%/94\% and CTK Recombilisa kit 94\%/97\% respectively.

Seasonal prevalence of leptospirosis in the rainy seasons was also noted in our study as the peak of seropositivity was observed between months of July to November in 2017. Rainy season leads to flooding and infestation with rodents, rats leading to transmission and perpetuation of the rodent -human infection cycle ${ }^{12,13}$. Males (77\%) showed higher positivity as compared to females $(23 \%)$ in our study owing to the more outdoor activities by men as compared to women. This observation correlates well with other similar studies $^{11,12}$.

The most common presenting feature was fever with myalgia and headache. This finding was consistent with other similar studies from India ${ }^{14}$. 
However, one such study also reported headache with fever as the most common symptom ${ }^{15}$. Out of the diagnosed patients, majority were treated for leptospirosis and showed significant improvement during the follow-up period. Typhoid was identified as the most common co-infection in these patients ${ }^{16,17}$. The higher rates of typhoid in these patients may be due to the common symptoms like fever, jaundice, liver enlargement which may also be suspected in leptospirosis. These infections sharing the same presenting features in the patient pose a challenge to the clinicians and require a high index of suspicion on his part ${ }^{17,18}$.

\section{CONCLUSION}

In our study we used ELISA to study the seroprevalence of leptospirosis in our hospital. ELISA can prove to be a very useful tool for diagnosis in resource-limited settings. We also compared two different ELISA kits to further strengthen our study. Both the ELISA kits showed comparable seroprevalence of $5.1 \%$. The present study gives a good idea on the seropositivity rate of Leptospirosis in this region of North India. Improvement in the socio-economic conditions, hygiene and sanitation has led to the decrease in seroprevalence rates of Leptospirosis. This neglected tropical disease must be dealt with high index of suspicion both in diagnosis and treatment as it may give rise to life-threatening complications.

\section{ACKNOWLEDGMENTS}

None.

\section{CONFLICT OF INTEREST}

The authors declare that there is no conflict of interest.

\section{AUTHORS' CONTRIBUTION}

SS performed literature search, data analysis, and first draft of the manuscript and figures. SSP and CS carried out the subsequent revisions of the manuscript, data analysis and final draft of the manuscript. HV contributed with laboratory work and investigations.

\section{FUNDING}

None.

\section{ETHICS STATEMENT}

Ethical clearance was taken from the institute' s ethics committee.

\section{DATA AVAILABILITY}

All datasets generated or analyzed during this study are included in the manuscript and/or the Tables.

\section{REFERENCES}

1. Heath CW Jr, Alexander AD, Galton MM. Leptospirosis in the United States. Analysis of 483 cases in man, 1949, 1961. N Engl J Med. 1965;273:915-922. https://doi. org/10.1056/NEJM196510212731706

2. Victoriano AF, Smythe LD, Gloriani Barzaga $N$, et al. Leptospirosis in the Asia Pacific region. BMC Infect Dis. 2009;9:147. https://doi.org/10.1186/1471-2334-9-147

3. Sethi S, Sharma N, Kakkar N, et al. Increasing trends of leptospirosis in Northern India: A clinic epidemiological study. PLoS Negl Trop Dis. 2010;4:e579. https://doi. org/10.1371/journal.pntd.0000579

4. Pappas G, Papadimitriou P, Siozopoulou V, et al. The globalization of leptospirosis: Worldwide incidence trends. Int J Infect Dis. 2008;12:351-357. https://doi. org/10.1016/j.ijid.2007.09.011

5. Levett PN. Leptospirosis. Clin Microbiol Rev. 2001;14:296-326. https://doi.org/10.1128/ CMR.14.2.296-326.2001

6. Singhal RL, Sood OP. Proceedings of the 3rd Round Table Conferences; 23 February 1998. Leptospirosis. New Delhi, India: Ranbaxy Science Foundation; 1998.

7. Chaudhry R, Saigal K, Bahadur T, et al. Varied presentations of leptospirosis: Experience from a tertiary care hospital in North India. Trop Doct. 2017;47:128-132. https://doi. org/10.1177/0049475516687431

8. Sethi S, Sood A, Pooja, et al. Leptospirosis in northern India: A clinical and serological study. Southeast Asian J Trop Med Public Health. 2003;34:822-825.

9. Chaudhry R, Das A, Premlatha MM et al. Serological \& molecular approaches for diagnosis of leptospirosis in a tertiary care hospital in North India: A 10-year study. Indian J Med Res. 2013;137:785-790.

10. Agrawal SK, Chaudhry R, Gupta N, et al. Decreasing trend of seroprevalence of leptospirosis at All India Institute of Medical Sciences New Delhi: 2014-2018. J Family Med Prim Care. 2018;7:1425-1428. https://doi.org/10.4103/ jfmpc.jfmpc_198_18

11. Garvey P, Connell J, O'Flanagan D et al. Leptospirosis in Ireland: Annual incidence and exposures associated with infection. Epidemiol Infect. 2014;142:847-855. https:// doi.org/10.1017/S0950268813001775

12. Sohail ML, Khan MS, Ijaz M et al.Seroprevalence and risk factor analysis of human leptospirosis in distinct climatic regions of Pakistan. Acta Trop. 2018;181:79-83. https:// doi.org/10.1016/j.actatropica.2018.01.021 
13. Tilahun Z, Reta D, Simenew K. Global epidemiological overview of leptospirosis. Int J Microbiol Res. 2013;4:9-15.

14. Muthusethupathi MA, Shivakumar S, Suguna R, et al. Leptospirosis in madras - A clinical and serological study. J Assoc Physicians India. 1995;43: 456-858.

15. Barua HC, Biswas D, Mahanta J. Clinico-epidemiological study on leptospirosis in certain parts of north-eastern region. J Commun Dis. 1999;31:201-2.

16. Patil VC, Patil HV, Agrawal V. Clinical profile and outcome of leptospirosis at tertiary care centre in western
Maharashtra. J Acad Med Sci. 2012;2:30-7. https://doi. org/10.4103/2249-4855.104013

17. Ittyachen AM, Krishnapillai TV, Nair MC, et al. Retrospective study of severe cases of leptospirosis admitted in the intensive care unit. J Postgrad Med. 2007;53:232-5. https://doi.org/10.4103/0022-3859.37510

18. Rao PS, Sridhar VS. Serological evidence in suspected cases of leptospirosis in and around Manipal. Indian J Med Microbiol. 2001;19:32. 